\title{
Robust Delegation Signcrypted Authentication Protocol against FHLR Attack in 3GPP Wireless Communications
}

\author{
Esam A. A. Hagras ${ }^{1}$, Maha Amer ${ }^{2}$ and, Hazem H. Aly ${ }^{3}$ \\ ${ }^{1}$ Faculty of Engineering, Alexandria University, Alexandria, Egypt \\ ${ }^{2,3}$ Arab Academy for Science and Technology and Maritime Transport, Cairo, Egypt. \\ ${ }^{1}$ esamhagras_2006@yahoo.com, ${ }^{2}$ maha_m.amer@hotmail.com, \\ ${ }^{3}$ hazemhali@gmail.com
}

\begin{abstract}
In this paper, we propose a new Robust Delegation Signcrypted Authentication Protocol (RD-SAP) against the False Home Location Register Attack in 3GPP. The proposed authentication protocol is based on Public Key Signcryption technique to solve the problem of FHLR attack on Tian F Lee protocol and which provides the user identity privacy, mutual authentication, nonrepudiation. This study also presents an enhanced protocol, which is not only has the same security properties as the original protocol, but also avoids the weakness in the original protocol. Therefore this scheme enjoys both computational and communicational efficiency.
\end{abstract}

Keywords: Robust delegation, Mobile authentication, Public key signcryption, False home location register.

\section{Introduction}

Portable Communication Systems (PCSs) [4] do not require any physical circuits between subscriber and service provided. PCSs technology allows users to carry portable communication devices that are low power, low cost, and small in size with mobile networking capabilities. Radio waves being transmitted in space make it easy for anyone to eavesdrop on the contents of communication, so there are more security and privacy threats than with wire line communication system. A secure communication system should possess four major features: secrecy, authenticity, integrity, and non-repudiation [5].

With the advancement of mobile technology, wireless networks have become widely available and interconnected. For allowing people to get connected seamlessly using their mobile devices without being limited by the geographical coverage of their own home networks, roaming services have been deployed, for example, GMS [6-9], 3GPP [10], and WLANs. A typical roaming scenario involves three parties: a roaming Mobile Station (MS), a Visited Location Register (VLR) and a Home Location Register (HLR). MS, who is a subscriber of HLR, is now in a network administered by VLR. There is a direct link between MS and VLR and another between VLR and HLR. But there is no link between MS and HLR. To prevent fraudulent use of services, user authentication is a mandatory requirement. The conventional way to perform user authentication is to let VLR contact HLR who acts as a guarantor for vouching that MS is a legitimate subscriber of it.

Public key cryptosystems have been used for mobile authentication in wireless networks [1], [13], [14], [15]. He et al. [15] used blind signature to design a privacy protection scheme for mobile stations; the scheme also provides $M S$ authentication and access authorization. Lee DOI : $10.5121 /$ ijdps.2011.2502 
and Yeh [1] proposed a trust delegation based scheme, where an $M S$, that is registered to a home location register $(H L R)$ or home network, proves its registration to a VLR (or serving network).

In this paper, we propose a new RDSA protocol against the False Home Location Register Attack in 3GPP. The proposed authentication protocol is based on Public Key Signcryption [11-12] technique to solve the problem of FHLR attack on Tian F. Lee protocol. Security analysis, storage capacity, computational cost and communicational overhead have been discussed for the proposed protocol.

The reminder of this paper is recognized as follows. Section 2 discusses the related work on the concept of delegation authentication protocols on 3GPP wireless communication systems. Section 3 reviews the concept of the enhanced authentication protocol of Tian-Fu Lee [12]. Section 4 describes the FHLR attack on the Tian-Fu Lee protocol. Section 5 presents our enhancement protocol. Section 6 proposes the security analysis. Finally Section 7 is the conclusion.

\section{Related Works}

Wireless communication systems [4] [16] provide mobile users with global roaming service. To support greater properties, numerous authentication approaches employ the public-key system to develop their protocols. For instance, long et al. [17] in 2004 presented a localized authentication protocol for inter-network roaming across wireless LANs. Lee et al. [18] in 2005 proposed a private authentication protocol to prevent the home location register (HLR) from eavesdropping on communication between the roaming station (MS) and the visited location register (VLR). However, due to hardware limitations, MS cannot support heavy encryption and decryption, and therefore wastes a lot of time in exponential computations.

In 2005, Lee and Yeh [1] presented the concept of delegation in wireless communication systems and proposed delegation based authentication protocol to solve the problem of data security, user privacy, computational loads and communicational efficiency in the system. Their protocol also adopted the public key cryptosystem to achieve the security requirements. To increase the communicational efficiency, and save authentication processes such that VLR does not need to contact HLR frequently, and a rapidly re-authenticate MS. Therefore, Lee protocol not only has a lower computational load for MS, but also provides greater security.

Although the protocol of Lee and Yeh exhibit non-repudiation in on line authentication process, it still has a weakness in off line authentication process. This weakness is that any legal VLR can forge authentication messages without the help of the mobile user. However, these forged messages are verified, and the mobile user cannot repudiate that he is the producer of these message. The malicious VLR can trick the HLR by these forged messages. That is, the protocol of Lee and Yeh does not have the property of non-repudiation in the off line authentication processes. Without the non repudiation property, a protocol may inspire a mobile user to deny that he has used services and refuse to pay, or inspire a services provider to overcharge a mobile user for services that he did not request [2].

In 2009, Tian F. Lee et al., enhanced Lee and Yeh protocol to solve the off line nonrepudiation problem by using the backward hash chain to ensure that the authentication messages in off line process cannot be forged. Although the protocol of Tian et al. solve the off line non-repudiation problem in Lee and Yeh protocol, it still has a weakness against False Home Location Register (FHLR) attack. In [3], FHLR attack on Lee and Yeh [1] protocol has 
been introduced. We adapt the FHLR attack given in [3] to attack Tian F. Lee et al protocol [2] which is an enhancement Lee and Yeh protocol.

\section{Review of Tian F. Lee et al protocol}

This section briefly reviews the enhanced delegation based authentication protocol of Tian F. Lee et al. [2] for PCSs, some notation should be explained here: $X \rightarrow Y: Z$, denotes that the sender $X$ sends a message $Z$ to a receiver $Y ; h(\cdot)$, denotes a one way hash function; $h^{2}($.$) ,$ denotes that the value is hashed twice; $n_{1} \| n_{2}$, denotes a concatenation of data $n_{1}$ and $n_{2} ; I D_{V}, I D_{H}$, denotes the identity of $V L R$ and $H L R$, respectively; $K_{H V}, K_{V H}$, denotes the secret key shared by VLR and HLR; $E_{k}[\bullet], D_{k}[\bullet]$ denotes a message encryption and decryption using a secret key $k$.

\subsection{Setup}

HLR generates parameters $p$ : a 512 bit prime; $q$ : a 160 bit prime factor of ( $p-1) ; g$ : an element where $g=z^{(p-1) / q} \bmod p$ and $z \in[1, p-1] ; x_{H L R}$ : a number less than $q$ as a HLR private key; $y_{H L R}: H L R$ public key certificates by Trusted Certificate Authority (TCA) where, $y_{H L R}=g^{x_{H L R}} \bmod p$. When user $M S$ registers in its $H L R, H L R$ create a proxy pair keys that contains a pseudonym are used to represent the real identity of $M S$ in the network. The relation between the pair keys and the corresponding real identity of $M S$ are protected in a secure database located in HLR. No one except HLR can obtain any information about the real identity of $M S$.

When $M S$ subscriber to his home system $H L R, H L R$ will generate random numbers $k$ and compute $K=g^{k} \bmod p$, and calculate $\sigma=x_{H L R}+k K \bmod q$, where, $\sigma$ is the secret key shared by $H L R$ and $M S$ and $K$ are the pseudonyms of $M S$. After that, the $M S$ will obtain a SIM card with its own key pair $(\sigma, K)$ from $H L R$. $M S$, generates random number " $n_{1}$ ", pre-computed a hash chain $h^{(1)}\left(n_{1}\right), h^{(2)}\left(n_{1}\right), \cdots, h^{(n+1)}\left(n_{1}\right)$ and stores them in its database, where $h^{(1)}\left(n_{1}\right)=h\left(n_{1}\right)$ and $h^{(i+1)}\left(n_{1}\right)=h\left(h^{(i)}\left(n_{1}\right)\right)$ for $i=1,2, \ldots, n$.

\subsection{On line authentication:}

Step 1: $M S \rightarrow V L R: K$. MS Sends its pseudonym $K$ to VLR

Step 2: VLR $\rightarrow M S: n_{2}, I D_{V}$

VLR Randomly generated $n_{2}$ (a number less than $q$ ) and sends $n_{1}, I D_{V}$ to $M S$.

Step 3: $M S \rightarrow V L R: r, s, K, N_{1}, I D_{H}, I D_{V}$

$M S$ Generates a random number $t$, picks $N_{1}=h^{(n+1)}\left(n_{1}\right)$ stored in its database, signs $N_{1}, n_{2}, I D_{V}$ and sends $r, s, K, N_{1}, I D_{H}, I D_{V}$ to $V L R$, where, $r, s$ are given by: $r=g^{t} \bmod p$

$$
s=\sigma * h\left(N_{1}\left\|n_{2}\right\| I D_{V}\right)+t * r \bmod q
$$


International Journal of Distributed and Parallel Systems (IJDPS) Vol.2, No.5, September 2011

Step 4: VLR $\rightarrow H L R:\left[N_{1}\left\|n_{2}\right\| K\right]_{K_{H V}}, I D_{H}, I D_{V}$

VLR checking if $g^{s}=\left(y_{H L R} K^{K}\right)^{h\left(n_{1} \mid n_{2} \| I D_{V}\right)} r^{r} \bmod p$, if the verifications are not achieved, the request is rejected; otherwise, $V L R$ sends $\left[N_{1}\left\|n_{2}\right\| K\right]_{K_{H V}}, I D_{H}, I D_{V}$ to $H L R$.

Step 5: HLR $\rightarrow \operatorname{VLR}:\left[\left[N_{1}, n_{3}, I D_{V}\right]_{\sigma}\left\|n_{2}\right\| l \| C_{1}\right]_{H V}, I D_{H}, I D_{V}$

$H L R$ decrypts the message $\left[N_{1}\left\|n_{2}\right\| K\right]_{K_{H V}}$ and obtains $K$ of each $M S$. If he successfully searches the corresponding $\sigma$ in its database according to $K$, then he computes $C_{1}=h\left(N_{1}\left\|n_{2}\right\| n_{3} \| \sigma\right), l=N_{1}$ where $n_{3}$ is a random number selected by $H L R$, and sends $\left[\left[N_{1}, n_{3}, I D_{V}\right]_{\sigma}\left\|n_{2}\right\| l \| C_{1}\right]_{K_{H V}}, I D_{V}, I D_{H}$ to $V L R$.

Step 6:VLR $\rightarrow M S:\left[N_{1}, n_{3}, I D_{V}\right]_{\sigma}, I D_{V}$

$V L R$ decrypts $\left[\left[N_{1}, n_{3}, I D_{V}\right]_{\sigma}\left\|n_{2}\right\| l \| C_{1}\right]_{K_{H V}}$ and obtains $\left[\left[N_{1}, n_{3}, I D_{V}\right]_{\sigma}, n_{2}, l\right.$ and $C_{1}$. Then he checks $n_{2}$ and $l$, sets the current session key $S K=C_{1}$ used by $V L R$ and $M S$, and forwards $\left[N_{1}, n_{3}, I D_{V}\right]_{\sigma}$ and $I D_{V}$ to $M S$. Finally, $M S$ decrypts $\left[N_{1}, n_{3}, I D_{V}\right]_{\sigma}$ and checks $N_{1}$ and computes the current session key $S K=C_{1}$.

\section{3 $i-t h$ Off line authentication}

$M S$ picks $h^{(n-i-1)}\left(n_{1}\right)$ stored in his database and sends $\left[h^{(n-i+1)}\left(n_{1}\right)\right]_{C_{i}}$ to $V L R$ for $i=1,2, \ldots, n$, where a predefined constant $n$ is the limited times of off line authentications. On receiving the authentication message from $M S, V L R$ checks whether $h\left(h^{(n-i-1)}\left(n_{1}\right)\right)$ and $l$ are equal, updates $l=h^{(n-1+1)}\left(n_{1}\right)$ and computes the session key $C_{i+1}=h\left(l, C_{i}\right)$. He also updates the count $i=i+1$ and checks $i<n$.

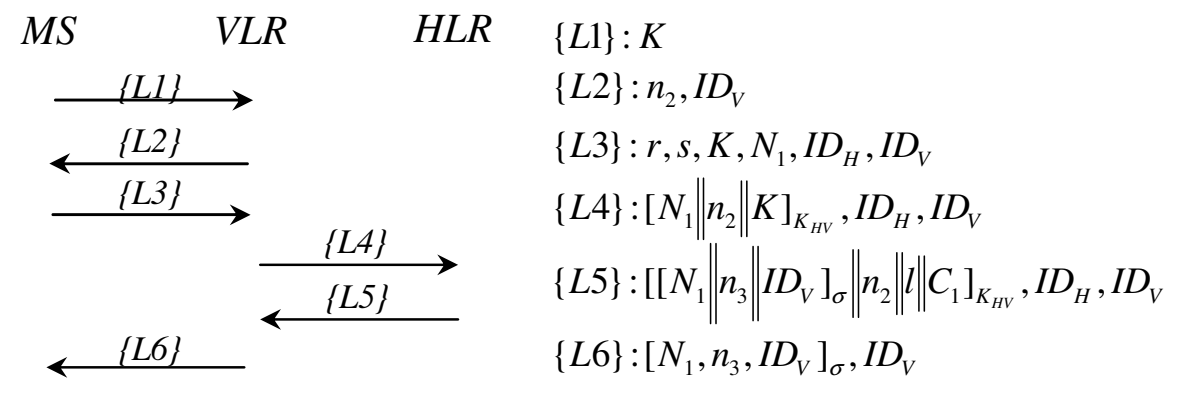

Fig. 1 Mobile Authentication Scheme of Tian F. Lee protocol [2]

\section{FHLR attack on Tian F. Lee et al protocol}


In Fig. 1 an attacker can first divert the $V L R$ to an $H L R$ under control of the adversary, and we denote this impersonated $H L R$ by FHLR with identification $I D_{F}$. The attacker modifies $I D_{H}$ in $\{L 3\}$ to $I D_{F}$. The modified message $\left\{L_{3}\right\}$ is defined in (1).

$$
\left\{L_{3}\right\}: r, s, K, N_{1}, I D_{F}, I D_{V}
$$

After the diversion, the attacker, that acts as a $V L R$, then obtains a session key $K_{(F, H)}$ with the legitimate $H L R$ of the $M S$ in question, and sends $\left\{L_{4}\right\}$ defined as in (2) instead of $\{L 4\}$ to the legitimate $H L R$.

$$
\left\{L_{4}\right\}:\left[N_{1}\left\|n_{2}\right\| K\right]_{K_{F H}}, I D_{H}, I D_{F}
$$

After the attacker receives $\left\{L_{5}\right\}$ (from $H L R$ ), which is defined as in (3), where $n_{3}$ is a random number selected by $H L R$, the attacker successfully obtains the session key $C_{1}$.

$$
\left\{L_{5}\right\}:\left[\left[N_{1}, n_{3}, I D_{V}\right]_{\sigma}\left\|n_{2}\right\| l \| C_{1}\right]_{K_{H F}}, I D_{H}, I D_{F}
$$

Let $K_{V F}$ be the session key between $V L R$ and FHLR, by following the protocol, after processing $\left\{L_{3}\right\}$, VLR can generate $\left\{\overline{L_{4}}\right\}$ as defined in (4) and sends it to FHLR that is under control of the attacker. FHLR that now acts as an HLR to the $M S$ in question can then reply to $V L R$ a newly composed $\left\{\overline{L_{5}}\right\}$ as defined in (5). This is straightforward since FHLR has the encrypted $\left[N_{1}, n_{3}, I D_{V}\right]_{\sigma}$ the random number $n_{2}$, the hashed value $l$ and the session key $C_{1}$ between $M S$ and $V L R$.

$$
\begin{aligned}
\left\{\overline{L_{4}}\right\}:\left[N_{1}\left\|n_{2}\right\| K\right]_{K_{V F}}, I D_{V}, I D_{F} \\
\left\{\overline{L_{5}}\right\}:\left[\left[N_{1}, n_{3}, I D_{V}\right]_{\sigma}\left\|n_{2}\right\| l \| C_{1}\right]_{K_{V F}}, I D_{H}, I D_{F}
\end{aligned}
$$

Now that $V L R$ and $M S$ follow the protocol and proceed to the remaining steps of the on-line and off-line authentication of Tian-Fu Lee. Figure 2 shows the messages used in this attack. This attack occurs because there is no security protection on $I D_{H}$ when it is sent through communication channels to $V L R$, so in equation (5) the attacker can obtain the session key $C_{1}$ since he has $K_{V F}$ and then the legitimate $H L R, V L R$ and $M S$ cannot know the fact that the $C_{1}$ is compromised. In our new protocol we enhance the authentication process of Tian-Fu Lee protocol by protecting the $I D_{H}$ by using a signcryption algorithm to ensure the data security, user privacy, computational load and communicational efficiency.

$$
\begin{aligned}
& \left\{L_{1}\right\}: K \\
& \left\{L_{2}\right\}: n_{2}, I D_{V} \\
& \left\{L_{3}\right\}: r, s, K, N_{1}, I D_{F}, I D_{V} \\
& \left\{\overline{L_{4}}\right\}:\left[N_{1}\left\|n_{2}\right\| K\right]_{K_{V F}}, I D_{V}, I D_{F} \\
& \left\{\overline{L_{5}}\right\}:\left[\left[N_{1}, n_{3}, I D_{V}\right]_{\sigma}\left\|n_{2}\right\| l \| C_{1}\right]_{K_{V F}}, I D_{H}, I D_{F} \\
& \text { MS VLR FHLR HLR }
\end{aligned}
$$

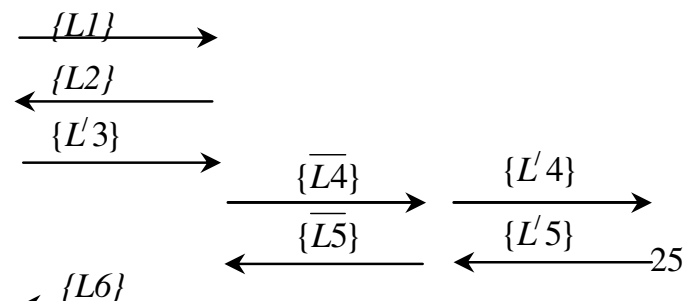




$$
\begin{aligned}
& \left\{L_{4}\right\}:\left[N_{1}\left\|n_{2}\right\| K\right]_{K_{V F}}, I D_{H}, I D_{F} \\
& \left\{L_{5}^{\prime}\right\}:\left[\left[N_{1}, n_{3}, I D_{V}\right]_{\sigma}\left\|n_{2}\right\| l \| C_{1}\right]_{K_{V F}} I D_{H}, I D_{F} \\
& \left\{L_{6}\right\}:\left[N_{1}, n_{3}, I D_{V}\right]_{\sigma}, I D_{V}
\end{aligned}
$$

Fig. 2, FHLR Attack on Mobile Authentication Scheme in [3]

\section{Proposed RDSA Protocol}

This section presents a new RDSA protocol that solves the problem of FHLR based on Tian$\mathrm{Fu}$ Lee and signcryption algorithm and then analysis its security and performance. In our protocol we employ a signcryption algorithm [12] to provide an efficient mobile authentication scheme and to solve the problem of FHLR which described in the previous section. Figure 3 illustrate our protocol which works as follow.

\subsection{Setup}

The setup phase is similar to that of the authentication protocol of Tian-Fu Lee [2]. HLR and $M S$ have their private/public key pairs $\left(x_{H L R}, y_{H L R}\right)$ and $(\sigma, K)$, respectively. The key pair $(\sigma, K)$ is also stored in $M S$ 's SIM card. Beside, $M S$ generates random number $n_{1}$, pre computes a hash chain $h^{(1)}\left(n_{1}\right), h^{(2)}\left(n_{1}\right), \ldots, h^{(n-1)}\left(n_{1}\right)$ and stores them in its database where $h^{(1)}\left(n_{1}\right)=h\left(n_{1}\right)$ and $h^{(i+1)}\left(n_{1}\right)=h\left(h^{(i)}\left(n_{1}\right)\right)$ for $i=1,2, \ldots, n$. Also we assume that $M S$ has chosen a random number $x_{M S}$ from $[1, \ldots ., q-1]$, and calculates its public number $y_{M S}=g^{x_{M S}} \bmod p$, also, VLR's chosen random number $x_{V L R}$ from $[1, \ldots ., q-1]$, and calculates its public number $y_{V L R}=g^{x_{V L R}} \bmod p$.

\subsection{On-line authentication:}

Step 1 MS $\rightarrow$ VLR: $K$.

$M S$ sends $K$ to $V L R$.

Step 2 VLR $\rightarrow \mathrm{MS}: I D_{V}, y_{V L R}$.

$V L R$ sends $I D_{V}$ and $y_{V L R}$ to $M S$.

Step $3 M S \rightarrow V L R: r, s, K, N_{1}, C_{H}, I D_{H}, I D_{V}$

$M S$ selects a random number $t$ from $[1, \cdots, q]$, picks $N_{1}=h^{(n+1)}\left(n_{1}\right)$ stored in its database, $\quad \operatorname{sign} N_{1}, y_{V L R}, I D_{V}$ and sends $r, s, K, N_{1}, E I D_{H}, E I D_{V}, I D_{H}, I D_{V}$ to $V L R$, where, $r, s$ and $E I D_{H}, E I D_{V}$ are given by:

$$
\begin{gathered}
\left(k_{1}, k_{2}\right)=h \operatorname{ash}\left(\left(\left(y_{V L R}\right)^{t}\right) \bmod p\right) \\
\left(E I D_{H}, E I D_{V}\right)=E_{k_{1}}\left[I D_{H} \| I D_{V}\right] \\
r=K_{k_{2}}\left(k_{1}, y_{V L R}, N_{1}, I D_{H}, I D_{V}\right) \\
s=\left(t /\left(r+\sigma_{H L R-M S}\right)\right) \bmod q
\end{gathered}
$$


International Journal of Distributed and Parallel Systems (IJDPS) Vol.2, No.5, September 2011

The output of the one-way hash is a binary number of at least 128 bits, which guarantees that, both $k_{1}$ and $k_{2}$ has at least 64 bits. The function $K_{k 2}(m)$ is a keyed hash algorithm for hashing a message ' $m$ ' under a key $k_{2}$. MS sends $r, s, K, N_{1}, E I D_{H}, E I D_{V}, I D_{V}$ and $I D_{H}$ to $V L R$.

Step 4 VLR $\rightarrow$ HLR $:\left[N_{1}\left\|y_{V L R}\right\| K\left\|I D_{H}\right\| I D_{V}\right]_{K_{H V}}, I D_{H}, I D_{V}$

1- VLR unsigncrypts $I D_{H}$ to get $\left(k_{1}, k_{2}\right)$ and checks the validity of its $r, s$.

2- VLR decrypts $\left(I D^{\prime}{ }_{H}, I D^{\prime}{ }_{V}\right)=D_{k_{1}}\left[E I D_{H} \| E I D_{V}\right]$

3-VLR computes $r^{\prime}=K H_{k_{2}}\left(k_{1}, N_{1}, y_{V L R}, I D_{H}^{\prime}\right)$ and checks if $r^{\prime}=r$. If $r^{\prime} \neq r$, reject and if $r^{\prime}=r, V L R$ sends $\left[N_{1}\left\|y_{V L R}\right\| K\left\|I D_{H}^{\prime}\right\| I D_{V}^{\prime}\right]_{K_{H V}}, I D_{H}, I D_{V}$ to $H L R$.

Theorem 1: If the signer $M S$ can strictly carry out above signcryption steps, the signcryption $(r, s)$ can pass the test of validity, and the specified receiver $V L R$ can also recover the original $H L R$ identity $I D_{H}$.

$$
\begin{aligned}
& \text { Proof: } \quad\left(k_{1}, k_{2}\right)=\operatorname{hash}\left(\left(\left(\left(y_{H L R} K^{K}\right) g^{r}\right)^{s \cdot x_{V L R}}\right) \bmod p\right) \\
& =\operatorname{hash}\left(\left(\left(\left(g^{x_{H L R}}\left(g^{k}\right)^{K}\right) \cdot g^{r}\right)^{s \cdot x_{V L R}}\right) \bmod p\right) \\
& =\operatorname{hash}\left(\left(\left(g^{x_{H L R}+k K} g^{r}\right)^{s \cdot x_{V L R}}\right) \bmod p\right) \\
& =\operatorname{hash}\left(\left(\left(g^{\sigma+r}\right)^{s \cdot x_{V L R}}\right) \bmod p\right) \\
& =\operatorname{hash}\left(\left(\left(g^{\sigma+r}\right)^{\frac{t}{(\sigma+r)} \cdot x_{V L R}}\right) \bmod p\right) \\
& =h a s h\left(\left(\left(g^{x_{V L R}}\right)^{t}\right) \bmod p\right) \\
& =h a s h\left(\left(y_{V L R}\right)^{t} \bmod p\right)
\end{aligned}
$$

Step $5 \mathrm{HLR} \rightarrow \mathrm{VLR}:\left[\left[N_{1}, n_{3}, I D_{V}\right]_{\sigma}\left\|y_{V L R}\right\| l\left\|C_{1}\right\| I D_{H} \| I D_{V}\right]_{K_{H V}}, I D_{H}, I D_{V}$

$H L R$ decrypts $\left[N_{1}\left\|y_{V L R}\right\| K\left\|I D_{H}\right\| I D_{V}\right]_{K_{H V}}$ and obtains $K$. If he successfully searches the corresponding $\sigma$ in its database according to $K$, then he computes $C_{1}=h\left(N_{1}\left\|y_{V L R}\right\| n_{3} \| \sigma\right)$, and $l=N_{1}$, where $n_{3}$ a random is number, and sends: $\left[\left[N_{1}, n_{3}, I D_{V}\right]_{\sigma}\left\|y_{V L R}\right\| l\left\|C_{1}\right\| I D_{H} \| I D_{V}\right]_{K_{H V}}, I D_{H}, I D_{V}$ to $V L R$.

Step $6 \mathrm{VLR} \rightarrow \mathrm{MS}:\left[N_{1}, n_{3}, I D_{V}\right]_{\sigma}, I D_{V}$

$V L R \quad$ decrypts $\left.\quad\left[N_{1}, n_{3}, I D_{V}\right]_{\sigma_{S}}\left\|y_{V L R}\right\| l \| C_{1}\right\rfloor_{K_{H V}}$ and obtains $\left[N_{1}, n_{3}, I D_{V}\right]_{\sigma}$, $y_{V L R}, l, I D_{H}$ and $C_{1}$. Then he checks $I D_{H}, y_{V L R}$ and $l$, stores $l$ in its database, sets the current session key $S K=C_{1}$ used by $V L R$ and $M S$, and forwards $\left[N_{1}, n_{3}, I D_{V}\right]_{\sigma}, I D_{V}$ 
International Journal of Distributed and Parallel Systems (IJDPS) Vol.2, No.5, September 2011

to $M S$. Finally $M S$ decrypts $\left[N_{1}, n_{3}, I D_{V}\right]_{\sigma}$, checks $N_{1}$ and computes the current session key $S K=C_{1}$.

\subsection{Off-line authentication}

The Off-line authentication phase is similar to that of the authentication protocol of Tian-Fu Lee. The proposed Robust Delegation-Based Authentication protocol is shown in figure (3).

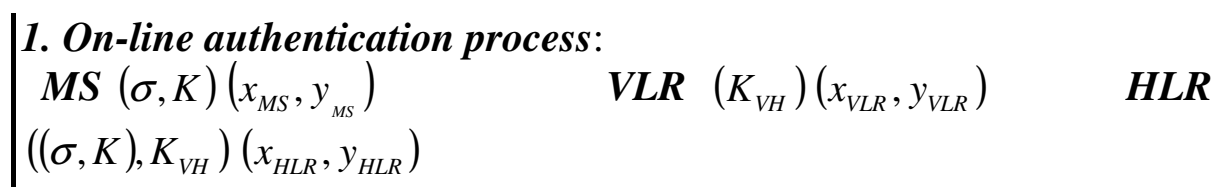

(0) Pre-compute and store

$h^{(1)}\left(n_{1}\right), h^{(2)}\left(n_{1}\right), \ldots, h^{(n-1)}\left(n_{1}\right)\left(=N_{1}\right)$

(1) $K$

(3.a) Calculate $\left(k_{1}, k_{2}\right)=\operatorname{hash}\left(y_{V L R}^{t} \bmod p\right)$

(2) $I D_{V}, y_{V L R}$

$$
\begin{aligned}
& \left(E I D_{H}, E I D_{V}\right)=E_{k_{1}}\left[I D_{H} \| I D_{V}\right] \\
& r=K H_{k_{2}}\left(k_{1}, y_{V L R}, N_{1}, E I D_{H}, E I D_{V}\right) \\
& s=t /(r+\sigma) \bmod q
\end{aligned}
$$

(3.b) $r, s, K, N_{1}, E I D_{H}, E I D_{V}, I D_{H}, I D_{V}$

(4.a) Check if:

$$
\begin{aligned}
& \left(k_{1}, k_{2}\right)=\operatorname{hash}\left(\left(\left(\left(y_{H L R} \cdot K^{K}\right) g^{r}\right)^{s \cdot x_{V L R}}\right) \bmod p\right) \\
& \left(I D_{H}^{\prime}, I D_{V}^{\prime}\right)=D_{k_{1}}\left[E I D_{H} \| E I D_{V}\right] \\
& r^{\prime}=K H_{k_{2}}\left(k_{1}, y_{V L R}, N_{1}, I D_{H}, I D_{V}\right) \\
& \text { (4.b) } \frac{\left[N_{1}\left\|y_{V L R}\right\| K\left\|I D_{H}\right\| I D_{V}\right]_{K_{H V}}, I D_{H}, I D_{V}}{\text { (5.a) Calculate: }}
\end{aligned}
$$

$C_{1}=h\left(N_{1}\|y\| n_{3} \| \sigma\right)$

$$
l=N_{1}
$$

(5.b) send:

$$
\begin{gathered}
{\left[\left[N_{1}, n_{3}, I D_{V}\right]_{\sigma}\left\|y_{V L R}\right\| l\left\|C_{1}\right\| I D_{H} \| I D_{V}\right]_{K_{H V}} \Psi D_{H}, I D_{V}} \\
\text { (6.a) Check } y_{V L R}, l, I D_{H} \text { and Store } l \\
\text { (6.b) }\left[N_{1}, n_{3}, I D_{V}\right]_{\sigma}, I D_{V}
\end{gathered}
$$


International Journal of Distributed and Parallel Systems (IJDPS) Vol.2, No.5, September 2011

(6.c) Check $N_{1}, I D_{V}$

(6.d) Calculate $S K: C_{1}=h\left(N_{1}\left\|y_{V L R}\right\| n_{3} \| \sigma\right)$

2. i-th Off-line authentication process:

$$
\begin{array}{cc}
\boldsymbol{M S}\left(C_{i}\right) & \boldsymbol{V L R}\left(C_{i}, l=h^{(n-i+1)}\left(n_{1}\right)\right) \\
\longrightarrow & \\
& \text { Check if } h\left(h^{(n-i)}\left(n_{1}\right)\right)=l \\
& \left.\left.\Rightarrow \text { Updates } l=h^{(n-i+1)}\left(n_{1}\right)\right]_{C_{i}}\right), \\
& S K: C_{i+1}=h\left(l, C_{i}\right) \text { and count } i=i+1<n
\end{array}
$$

Fig. 3 Proposed Robust Delegation Signcrypted Authentication Protocol

\section{Security Analysis}

The analysis of our protocol is similar to those of the protocol of Tian-Fu Lee et al, in offline authentication processes, and therefore is not presented herein.

\subsection{User Identity Privacy}

In our protocol $M S$ has one private/public key pair $(\sigma, K)$. The real identity of $M S$ is not transmitted over the entire network for authentication purposes. Because we use pseudonym $K$ generated by $H L R$ in the registration phase to represent the identity of $M S$ in the network, no one except $H L R$ can obtain any information about the identity on MS. Also for the private/public number pair $\left(x_{M S}, y_{M S}\right)$, MS sends its public number $y_{M S}$ to $V L R$ in the registration phase and uses the generated random number $t$ with the private number $x_{M S}$ to $\operatorname{sign} N_{1}, y_{V L R}$ and $I D_{H}$, where $r=K H_{k 2}\left(k_{1}, y_{V L R}, N_{1}, I D_{H}\right)$ and $s=t /(r+\sigma) \bmod q$. Hence our new protocol provides strong user identity privacy.

\subsection{Nonrepudiation}

In our protocol $M S$ sends the signcrypted text $(r, s, c)$ to $V L R$ and then $V L R$ unsigncrypt and verify this text. VLR accept this text if $r^{\prime}=K H_{k 2}$ and $\left(I D^{\prime}{ }_{H}, I D^{\prime}{ }_{V}\right)=D_{k_{1}}\left[E I D_{H} \| E I D_{V}\right]$, so, the attacker cannot forge it since $k_{1}$ and $k_{2}$ depend on " $t$ " which is randomly chosen by $M S$. Therefore our protocol guarantees that $M S$ generates the authentication message in online authentication processes.

\subsection{Mutual Authentication}

In the online authentication process, $V L R$ authenticates $M S$ by verifying the signcrypted text $r^{\prime}=K H_{k_{2}}\left(k_{1}, y_{V L R}, N_{1}, I D_{H}, I D_{V}\right)$ and $\left(I D^{\prime}{ }_{H}, I D^{\prime}{ }_{V}\right)=D_{k_{1}}\left[E I D_{H} \| E I D_{V}\right]$ in step 4, and authenticate $H L R$ by checking $n_{2}$ and $I D_{H}$ of $\left[\left[N_{1}, n_{3}, I D_{V}\right]_{\sigma}\left\|y_{V L R}\right\| l\left\|C_{1}\right\| I D_{H} \| I D_{V}\right]_{K_{H V}}$ in step 6, respectively. MS authenticates $V L R$ and $H L R$ by checking $N_{1}$ of $\left[N_{1}, n_{3}, I D_{V}\right]_{\sigma}, I D_{V}$ from $V L R$ 
in step 6. HLR authenticates $V L R$ by checking $K$ and $I D_{H}$ of $\left[N_{1}\left\|y_{V L R}\right\| K\left\|I D_{H}\right\| I D_{V}\right]_{K_{H V}}$ in step 5, and authenticates $M S$ through $V L R$ authenticating $M S$ in step 4, respectively. So, our protocol provides mutual authentication.

\subsection{Computational Cost and Communicational Overhead}

In this section we calculate the communicational cost and communicational overhead in each step of our scheme (in online authentication process) as seen in table I. Here we assume that $n=5$, so $N_{1}=h^{(4)}\left(n_{1}\right)$ since $N_{1}=h^{(n-1)}\left(n_{1}\right)$.

Table 1: Computational Cost and Communicational Overhead of our protocol

\begin{tabular}{|l|l|l|}
\hline \multicolumn{1}{|c|}{ Message transmission } & \multicolumn{1}{c|}{ Computational Cost } & \multicolumn{1}{c|}{ Communication Overhead } \\
\hline (0) $M S$ pre-compute and store $N_{1}$ & $n * \mathrm{HASH}$ & 0 \\
\hline (1) from $M S$ to $V L R$ & 0 & $512 * 1=64$ bytes \\
\hline (2) from $V L R$ to $M S$ & 0 & $512+32=68$ bytes \\
\hline (3) from $M S$ to $V L R$ & $\begin{array}{l}\text { HASH=2, DIV=1, ADD }=1, \\
\text { ENC=1, EXP=1 }\end{array}$ & $160 * 3+512+128+64=148$ bytes \\
\hline (4) from $V L R$ to $H L R$ & $\begin{array}{l}\text { HASH=2, MUL }=2, \mathrm{EXP}=2, \\
\text { DEC=1, ENC }=1\end{array}$ & $160+512 * 2+32 * 4=164$ bytes \\
\hline (5) from $H L R$ to $V L R$ & HASH=5, ENC $=2$ & $160 * 4+32 * 5+512=164$ bytes \\
\hline (6) from $V L R$ to $M S$ & HASH $=1, \mathrm{ENC}=1$ & $160 * 2+32 * 2=48$ bytes \\
\hline
\end{tabular}

$\mathrm{EXP}=$ modulo exponentiation, $\mathrm{HASH}=$ one-way or keyed hash, $\mathrm{DIV}=$ modulo division, $\mathrm{MUL}=$ modulo multiplication, $\mathrm{ADD}=$ modulo addition, $\mathrm{ENC}=$ encryption using private key, $\mathrm{DEC}=$ decryption using private key.

\subsection{Storage Capacity}

Storage capacity should be taken into account when designing security protocols for mobile network environments since the mobile equipment has limited storage capacity. Considering the example we take in figure 2, the mobile station should store the parameters $p$, $q, K=g^{k} \bmod p, \sigma=x+k K \bmod q, N_{1}, y_{V L R}, t, I D_{H}, I D_{V}, r=K H_{k_{2}}\left(k_{1}, y_{V L R}, N_{1}, I D_{H}, I D_{V}\right)$, $s=\left(t /\left(r+\sigma_{H L R-M S}\right)\right) \bmod q,\left(E I D_{H}, E I D_{V}\right)=E_{k_{1}}\left[I D_{H} \| I D_{V}\right], x_{M S},\left(k_{1}, k_{2}\right)=h a s h\left(y_{V L R}^{t} \bmod p\right)$, $y_{M S}=g^{x_{M S}} \bmod p, y_{V L R}=g^{x_{V L R}} \bmod p$, where $p$ is a 512 bit prime number, $q$ is a 160 bit prime factor of $p-1, N_{1}$ is a hash function of $160 \mathrm{bit}, x_{M S}$, and $t$ are numbers less than $q, r$ is a Secure Hash Algorithm-1 (SHA-1) of 160 bit, $C_{H}=\left(E I D_{H}, E I D_{V}\right)$ is 128 bit, the length of $I D_{H}$ is 32 bit, the length of $I D_{V}$ is 32 bit, and the output of the one-way hash is a 512 bit, which guarantees that both $k_{1}$ and $k_{2}$ have 256 bit where $\left(k_{1}, k_{2}\right)=\operatorname{hash}\left(y_{V L R}^{t} \bmod p\right)$.

Therefore, the total length of $\left(q, \sigma, N_{1}, t, r, s, x_{M S}, K, y_{V L R}, y_{M S}, p, k_{1}, k_{2}, I D_{V}, I D_{H}, C_{H}\right)$ is given by: $160 * 8+512 * 5+32 * 2+128=4032$ bit $=504$ bytes. The currently used SIM 
International Journal of Distributed and Parallel Systems (IJDPS) Vol.2, No.5, September 2011

card consists of $16 \mathrm{k}$ bytes of ROM, 256 bytes of RAM, and $8 \mathrm{k}$ bytes of Electrically Erasable Programmable ROM (EEPROM) [1]. In summary, the capacity of EEPROM is large enough to accommodate the above parameters of our scheme.

\section{Conclusions}

This investigation addresses the weakness of the enhanced delegation-based authentication protocol raised by Tian-Fu Lee, which cannot solve the problem of FHLR in online authentication process. Therefore, a new Robust Delegation Signcrypted Authentication Protocol (RD-SAP) against the False Home Location Register Attack in 3GPP is presented. The proposed authentication protocol is based on Public Key Signcryption technique and which provides the user identity privacy, mutual authentication, nonrepudiation. This study also presents an enhanced protocol, which is not only has the same security properties as the original protocol, but also avoids the weakness in the original protocol. Therefore this scheme enjoys both computational and communicational efficiency.

\section{REFERENCES:}

[1] W.-B. Lee and C.-K. Yeh, "A New Delegation-based Authentication Protocol for use in Portable Communication Systems," IEEE Trans. Wireless Commun., vol. 4, NO. 1, pp. 57-64, Jan. 2005.

[2] Tian-Fu Lee, Shu-Hui Chang, Tzonelih Hwang, and Song-Kong Chong, "Enhanced DelegationBased Authentication Protocol for PCSs", IEEE Trans. Wireless Commun., vol. 8, NO. 5, pp. 2166-2171, May 2009.

[3] Caimu Tang, Dapeng Oliver Wu, "An Efficient Mobile Authentication Scheme for Wireless Networks”, IEEE Trans. Wireless Commun., vol. 7, NO. 4, pp. 1408-1416, April 2008.

[4] H.-Y. Lin, "Security and authentication in PCS," Comput. Elect. Eng., vol. 25, no. 4, pp. 225-248, 1999.

[5] H.-Y. Lin and L. Harn, "Authentication protocols with non-repudiation services in personal communication systems," IEEE Commun. Lett., vol. 3, no. 8, pp. 236-238, Aug. 1999.

[6] M. Rahnema, "Overview of the GSM system and protocol architecture," IEEE Commun. Mag., pp. 92-100, Apr. 1993.

[7] A. Merotra and L. Golding, "Mobility and security management in the GSM system and some proposed future improvements," Proc. IEEE, vol. 86, pp. 1480-1496, July 1998.

[8] T.-F. Lee, C.-C. Chang, and T. Hwang, "Private authentication techniques for the global mobility network," Wireless Personal Commun., vol. 35, no. 4, pp. 329-336, Dec. 2005.

[9] K. Al-Tawill, A. Akrami, and H. Youssef, "A new authentication protocol for GSM networks," in Proc. 23rd Annual IEEE Conf. Local Comput. Networks, pp. 21-30, 1998.

[10] Nieme, Vand Nyberg K., "UMTS Security”, Nokia Research Center, Finland.

[11] Zheng, Y, "Digital signcryption or how to achieve cost (signature \& encryption) $<<$ cost (signature) + cost (encryption)". In Advances in Cryptology - CRYPTO'97 (Berlin, New York, Tokyo, 1997) vol. 1294 of Lecture Notes in Computer Science Springer-Verlag pp. 165-179, 1997.

[12] Zheng, Y, "Signcryption and Its Applications in Efficient Public Key Solutions" Monash University, McMahons Road, Frankston, Melbourne, VIC 3199, Australia.

[13] D. Samfat, R. Molva, and N. Asokan, "Untraceability in Mobile Networks", in Proc. of International Conference on Mobile Computing and Networking, 1995, P26-36. 
International Journal of Distributed and Parallel Systems (IJDPS) Vol.2, No.5, September 2011

[14] M. J. Beller, L.-F, Chang, and Y. Yacobi, "Privacy and Authentication on a Portable Communication System”, IEEE J. Select Areas Commun., vol. 11, NO. 6, pp. 821-829, Aug. 1993.

[15] Q. He, D. Wu, and P. Khosla, "Quest for Personal Control Over Mobile Location Privacy”, IEEE Commun. Mag., vol. 42, no. 5, pp. 130-136, 2004.

[16] A. Merotra and L. Golding, "Mobility and security management in the GSM system and some proposed future improvements," Proc. IEEE, vol. 86, pp. 1480-1496, July 1998.

[17] M. Long, C.-H. Wu, and J. D. Irwin, "Localised authentication for internetwork roaming across wireless LANs," IEE Proc. Commun., vol. 151, no. 5, pp. 496-500, Oct. 2004.

[18] T.-F. Lee, C.-C. Chang, and T. Hwang, "Private authentication techniques for the global mobility network," Wireless Personal Commun., vol. 35, no. 4, pp. 329-336, Dec. 2005.

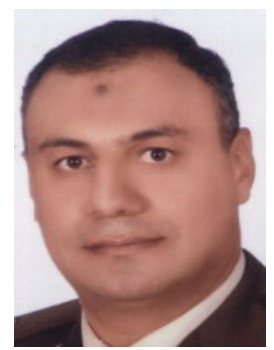

Esam A. A. HAGRAS received the B.S. degrees in Electrical Engineering from faculty of engineering, Alexandria Univ., Egypt, in 1994, M.S. degrees in Electrical Engineering from Mansoura Univ., Egypt, in 2001, respectively. During 2005-2007, he was on in Dept., of Electrical Engineering, faculty of engineering, Alexandria Univ. In Dec. 2007, he got the $\mathrm{PhD}$ degree in information security and communications.

His research interests in the field of information and multimedia security, chaotic cryptography, Hardware implementation of encryption algorithms on FPGA , data compression, digital image watermarking, communication and wireless sensor network security. He has published more than twenty papers on security and communications.

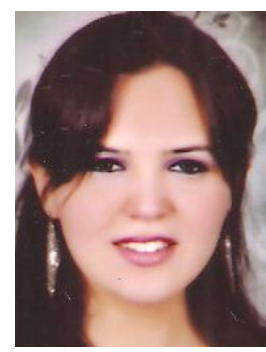

Maha Amer is currently a M.S. student in Department of Communications and Electronics at Arab Academy of Science and Technology and Maritime Transport. She obtained her B.S. degree in 2006 from faculty of engineering Banha University, Egypt. Her research interests in Security, Wireless Communication Systems, Next Generation of Mobile Communication Systems, and authentication and key management's techniques in $3 \mathrm{G}$ and UMTS networks.

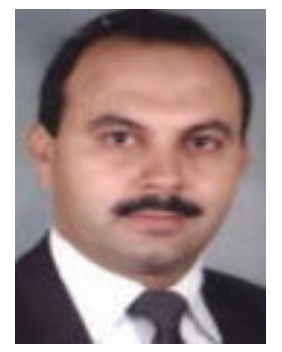

Hazem H. Ali - Professor and Chairman of Communication Department, Arab Academy of Science \& Technology and Maritime Transport, Egypt. He received the B.S. degrees in Electrical Engineering from faculty of engineering, Kuwait Univ., Kuwait, in 1987; M.S. and PhD degrees in Electrical Engineering from George Washington University, USA, in 1993 VLSI Systems and circuits.

His research interests in the field of VLSI Systems, MEMs, communication and wireless sensor network security. 